\title{
Student's Corner-3
}

\author{
Amar Patnaik' Dayasagar Rao Vala² \\ ${ }^{1}$ Sunshine Hospital, Hyderabad, Telangana, India \\ 2KIMS Hospital, Hyderabad, Telangana, India
}

Ind J Car Dis Wom 2021;6:68-70.

\section{Q. Parasternal Heave in Differentiating Volume Overload and Pressure Load on Right Ventricle}

\section{Explanation by Prof. Patnaik}

With each ventricular systole the left lower costal cartilages along with the attached part of sternum get elevated due to the hypertrophied and dilated right ventricle. The inspection and palpation of this phenomenon in supine position has become a standard method of clinical cardiac evaluation. ${ }^{1,2}$ Appreciation of this lift/heave at the left parasternal area is not as precise as the cardiac ape ${ }^{3}$ however, clinicians have learned to roughly quantify the right ventricular systolic pressure and volume changes.

It is most conveniently graded (All India Institute of Medical Sciences [AIIMS] gradation) as follows:

- 1/3 (Grade 1): visible but not palpable

- 2/3 (Grade 2): visible and palpable but obliterable

- 3/3 (Grade 3): visible and palpable but not obliterable

In the presence of significant right ventricular pressure overload the palpation of the left parasternal area will reveal a sustained left parasternal lift., ${ }^{4,5}$ There may be associated prominent "a" wave in jugular venous pressure (JVP) and abnormal epigastric and subxiphoid pulsations. Common causes of raised right ventricle (RV) pressure are pulmonary hypertension and severe pulmonary stenosis. In RV volume overloaded states, the left parasternal impulse is appreciable but not sustained. Atrial septal defect (ASD) with significant left to right shunt can have an associated tricuspid mid-diastolic flow murmur. The ASD or severe tricuspid or pulmonary regurgitation cause RV volume overload.

Large left atrial enlargement in severe mitral regurgitation sometimes produces left parasternal lift but it is mostly confined to late systole. Similarly, a dilated left ventricle (LV) that is rotated counterclockwise in severe AR can also cause parasternal lift. In children and thin adults, a parasternal
Address for correspondence M. Jyotsna, MD, DM, Department of Cardiology, Nizam's Institute of Medical Sciences, Punjagutta, Hyderabad, Telangana 500082, India (e-mail: janaswamyjyotsna@gmail.).

heave (PSH)-like chest motion may be appreciated without any heart disease.

\section{Explanation by Prof. Dayasagar Rao}

Parasternal Pulsations (Left)-Clinical Implications

In situs solitus state the most anterior structure of the heart is the right ventricle which is just below the anterior chest wall.

In patients with RV volume overload like ostium secundum ASD with large L-R shunt (and no pulmonary hypertension $[\mathrm{PH}]$ ) or low pressure tricuspid regurgitation (TR) there will be vigorous pulsation in intercostal spaces (3rd, 4th, and 5th) of left parasternal region consequent to dilation of RV. These pulsations are present over RV inflow (5th space) and $\mathrm{RV}$ outflow (3rd and 4th interspace) but no significant, sustained lift of precordium.

In some clinical situations RV outflow pulsations without inflow pulsation are seen, classically in Ebstein's anomaly of tricuspid valve (TV) where the inflow of RV is atrialized and in patients with RV and in endomyocardial fibrosis (EMF) where inflow is fibrosed plastering the posterior leaflet to TV.

In parasternal heave, unlike in the parasternal pulsation, precordium is lifted above the chest wall and sustained for significant time ( $>50 \%$ systole) and is characteristically seen in pressure overloaded RV, like in pulmonary hypertension and right ventricular outflow tract (RVOT) obstruction. The parasternal heave is graded (arbitrarily) as follows:.

- Grade I: visible, barely palpable

- Grade II: visible, palpable, but can be obliterated by counter-pressure of hand

- Grade III: parasternal heave that cannot be obliterated by counter-pressure (mild)

Occasionally in severe mitral regurgitation systolic expansile impulse of LA can be felt over left parasternal area even though LA is the most posterior structure (parasternal heave is a transmitted pulsation). This LA lift is better palpated in the upper parasternal area and occurs in later part of systole in contrast to early systolic lift of RV pressure overload. published online April 9, 2021
DOI https://doi.org/

10.1055/s-0041-1728181
(C)2021. Women in Cardiology and Related Sciences.

This is an open access article published by Thieme under the terms of the Creative Commons Attribution-NonDerivative-NonCommercial-License, permitting copying and reproduction so long as the original work is given appropriate credit. Contents may not be used for commercial purposes, or adapted, remixed, transformed or built upon. (https://creativecommons.org/licenses/by-nc-nd/4.0/).

Thieme Medical and Scientific Publishers Pvt. Ltd. A-12, 2nd Floor, Sector 2, Noida-201301 UP, India 


\section{Q. Concept of Hangout Interval and Its Influence on Split S2 in Physiological and Pathological Conditions}

\section{Explanation by Prof. Patnaik}

At the end of ejection of blood, that is, when the pressures in the ventricle and the great artery crossover), the semilunar valves are expected to close promptly. In reality, this mechanical event occurs with a slight time delay at point called the incisura. The hangout time is the interval between the end of ventricular ejection (when the ventricular pressure crosses over the pressure in the respective great artery) and the actual closure of the semilunar valve (-Fig. 1). This term was coined by Shaver and associates who were convinced that it represents impedance of the vasculature. The hangout interval is longer in the pulmonary circulation (60-70 milliseconds) than in the systemic side (15-30 milliseconds). The compliance of aorta and the pulmonary artery also influence the hangout interval. ${ }^{6}$

\section{Second Heart Sound Split in Health}

The A2 and P2 are appreciated as two separate sounds (40-60 milliseconds apart) during inspiration best heard at the pulmonary area. ${ }^{7}$ In expiration they move too close (10 milliseconds) to be appreciated as two separate sounds. The most accepted explanation is that during inspiration, pulmonary vasculature expands, the impedance in pulmonary vasculature decreases, and the pulmonary hangout interval further widens. Another explanation is increased inspiratory right ventricular venous return leads to prolonged RV mechanical systole (prolonged ejection time) leading to a delayed P2 component. The third explanation is some contribution of earlier A2 due to lesser preload to left ventricle and shorted LV mechanical systole in inspiration (Harris and Sutton). The difference in the hangout intervals in the two sides determines the splitting in the second heart sound., ${ }^{8,9}$

\section{Second Heart Sound in ASD and PS}

Wide and fixed split second heart sound is very characteristic clinical finding in ASD with significant left to right shunt. ${ }^{10,11}$ The split is said to be wide if the two components

Hangout interval in pulmonary circulation

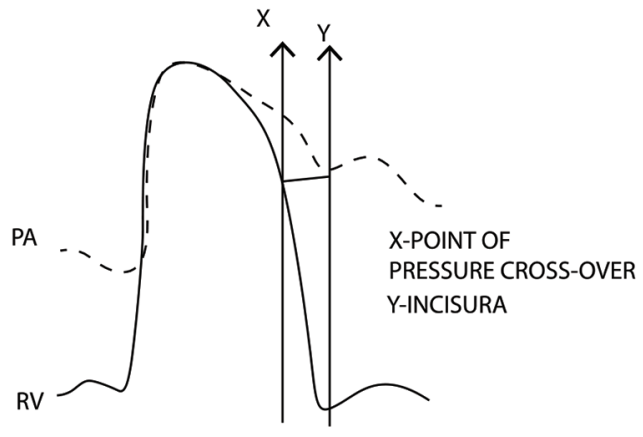

Hangout interval is the duration from point $X$ to point $Y$

Fig. 1 Hangout interval in pulmonary circulation. are heard in both phases of respiration. In ASD the increased right-sided flow (leading to increased hangout interval) causes delayed P2 and a wide split second heart sound. But on inspiration there is no further separation and hence it is called fixed. Shaver et al observed that increased hangout interval is the major mechanism that explains the wide split second heart sound in ASD in sinus rhythm with normal pulmonary pressure. In hyperkinetic pulmonary hypertension the hangout interval narrows, and right ventricular electromechanical interval decides the split. In PS, there is prolonged $\mathrm{RV}$ systole and prolonged ejection time and mechanical delay in closure of pulmonary valve. This leads to wide split second sound. However, second heart sound split is not fixed as the respiratory variations in venous return to the ventricles continue to occur.

\section{Second Heart Sound ASD with Pulmonary Hypertension}

The pulmonary vascular resistance is increased in pulmonary hypertension and it reduces the right sided hangout interval. ${ }^{12}$ The net result is narrow split second heart sound with loud P2 component or a single second heart sound. The fixity of the spit is retained.

\section{Second Heart Sound in VSD}

In small VSD the hemodynamics will not alter the hangout interval and the behavior of the second heart sound will be like in healthy individual. In large VSD the pulmonary hypertension is invariable and there is shortened right sided hangout interval. There can be shortened left-sided hangout interval due to earlier completion of LV ejection. This results in the two components of second heart sound to come close to each other and S2 may be appreciated as a single sound. Similar changes are also observed in hypertensive PDA. In Eisenmengerized VSD the hangout intervals of both circulations may equalize and a single second heart sound may be heard.

\section{Second Heart Sound Severe Aortic Stenosis}

Reverse split of second heart sound may be heard in some cases of severe AS due to prolonged LV ejection time and the increased aortic hangout interval.

\section{Explanation by Prof. Dayasagar Rao}

Hangout interval is time interval between the end of ventricular ejection and the closure of the semilunar valve, during which time, there is transient flow reversal in great vessels (aorta/pulmonary artery) facilitating closure of semilunar valves.

Timing of flow reversal is influenced by two factorscapacitance of circulation and resistance offered to forward flow (PVR-SVR) and compliance of great vessels. Normally pulmonary vascular resistance in much lower than systemic vascular resistance (PVR/SVR ratio: 0.25 or less), and PA is more compliant than aorta, both of which combine to have longer hangout interval (60-80 milliseconds) on right side, compared with left side (15-30 milliseconds). This difference in hangout interval contributes to degree of splitting of the second heart sound (A2-P2 intervals) as 
the electromechanical systole of both ventricles is nearly equal. Other hemodynamic factors that influence the A2P2 interval are duration and difference in electromechanical systole of ventricles and ventricular systolic function.

In $\mathrm{PH}$, the A2-P2 interval is short because hangout interval on right side is short due to increased PVR. The wide A2-P2 interval in idiopathic dilation of pulmonary artery (IDPA) is due to increase of hangout interval on right side, consequent to dilated and compliant main pulmonary artery (MPA). Persistent wide split second sound in ASD even after closure is explained by dilated PA causing increased hangout interval on right side.

\section{Conflict of Interest}

None declared.

\section{References}

1 Bethell HJN, Nixon PGF. Examination of the heart in supine and left lateral positions. Br Heart J 1973;35(9):902-907

2 Craige K, Inspection and palpation of the precordium. In: Hurst JW, et al, eds. The Heart. 5th ed. New York, NY: McGraw-Hill; 1982:199-202
3 Eilen SD, Crawford MH, O'Rourke RA. Accuracy of precordial palpation for detecting increased left ventricular volume. Ann Intern Med 1983;99(5):628-630

4 Hurst JW, Schlant RC, Examination of the Heart: III, Inspection and Palpation of the Anterior Chest. New York, NY: American Heart Association; 1972

5 Perloff JK, Physical Examination of the Heart and Circulation. Philadelphia, PA: WB Saunders; $1982 ; 130-170$

6 Klabunde RE, Cardiovascular Physiology Concepts. Philadelphia, PA: Lippincott Williams and Wilkins; 2012

7 Leatham A. The second heart sound: key to auscultation of the heart. Acta Cardiol 1964;19:395-416

8 Shaver JA. Clinical implications of the hangout interval. Int J Cardiol 1984;5(3):391-398

9 Loknath S, Rao VDS. Second heart sound. I J Car Dis Wom 2020; 5:76-86

10 Harris A, Sutton G. Second heart sound in normal subjects. Br Heart J 1968;30(6):739-742

11 Shafter HA. Splitting of the second heart sound. Am J Cardiol 1960;6:1013-1022

12 O'Toole JD, Reddy PS, Curtiss EI, Shaver JA. The mechanism of splitting of the second heart sound in atrial septal defect. Circulation 1977;56(6):1047-1053 